\title{
Linear active disturbance rejection control of the hovercraft vessel model
}

\author{
R. Morales, H. Sira-Ramírez, J.A. Somolinos
}

\begin{abstract}
A B S T R A C T
A linearizing robust dynamic output feedback control scheme is proposed for earth coordinate position variables trajectory tracking tasks in a hovercraft vessel model. The controller design is carried out using only position and orientation measurements. A highly simplified model obtained from flatness considerations is proposed which vastly simplifies the controller design task. Only the order of integration of the input-to-flat output subsystems, along with the associated input matrix gain, is retained in the simplified model. All the unknown additive nonlinearities and exogenous perturbations are lumped into an absolutely bounded, unstructured, vector of time signals whose components may be locally on-line estimated by means of a high gain Generalized Proportional Integral (GPI) observer. GPI observers are the dual counterpart of GPI controllers providing accurate simultaneous estimation of each flat output associated phase variables and of the exogenous and endogenous perturbation inputs. These observers exhibit remarkably convenient self-updating internal models of the unknown disturbance input vector components. These two key pieces of on-line information are used in the proposed feedback controller to conform an active disturbance rejection, or disturbance accommodation, control scheme. Simulation results validate the effectiveness of the proposed design method.
\end{abstract}

\section{Introduction}

Over the last two decades, a great amount of research has been conducted regarding the regulation marine control systems. They are employed in applications such as dredging operations, recovery of lost man-made objects, cable laying operations, towing operations, military operations, among others (Yuh, 2000; Fossen, 1994, 2011). These applications typically require the accurate tracking of trajectories thus implying the need for high performance controllers. Although ships are usually fully actuated, several other vessels are under-actuated. Control strategies for the under-actuated ship models cannot be asymptotically stabilized by continuous timeinvariant feedback control laws (Brockett, 1983). In Kaminer et al. (1998) is proposed a gain-scheduled controller to track reference trajectories in the inertial reference frame. The stabilization problem towards a desired equilibrium is treated in Reyhanoglou (1997). A time-varying feedback control law is proposed in Petersen and Egeland (1996), achieving exponential state stabilization. In Lebefer et al. (2003), a theoretical and experimental result is developed using Lyapunov-based controllers. In Behal et al. (2002) is presented a transformation of the error dynamics into a skew-symmetric form and achieves practical convergence. High-frequency feedback control signals, in combination with averaging theory and back-stepping, have been proposed in Pettersen and Nijmeijer (1998) to achieve practical stabilization of the ship toward a desired equilibrium. They were also extended for trajectory tracking tasks. In Wondergem et al. (2011) is presented an observer-controller scheme which takes into account the complete model dynamics, including Coriolis and centripetal forces and nonlinear damping, and results in a semi-globally uniformly stable closed loop system. Other recent approaches are optimal control (Prasanth Kumar et al., 2005), robust trajectory control based on direct estimation of system dynamics (Prasanth Kumar et al., 2007) or backstepping techniques (Repoulias and Papadopoulos, 2007), among others.

The hovercraft model used here is based on the work of Fantoni et al. (2000), where the vessel's dynamics are derived on the basis of the under-actuated ship model extensively studied in Fossen (1994). Planning and control of hovercraft systems can be considerably simplified by using the differential flatness property (Sira-Ramírez and Aguilar, 2000; Sira-Ramírez, 2002), obtained by specific design conditions. Differential flatness was proposed and developed in Fliess et al. (1995) (see also the book by 
Sira-Ramírez and Agrawal, 2004 for diverse engineering applications of this concept). In this paper, we propose a linearizing, global, approach to the robust output feedback controller design for output trajectory tracking tasks in the earth coordinate position variables of a hovercraft vessel. The solution is carried out with position and orientation measurements alone. The linear observer-based controller design approach, presented here, is most suitable for the ubiquitous class of differentially flat systems. The proposed observer based control approach, called Generalized Proportional Integral (GPI) observer-based control (Fliess et al., 2002), rests on using highly simplified models (defined in Fliess and Join, 2009), on the input-to-flat output models derived from the flatness property. The perturbation input lumps both external disturbance inputs and state-dependent nonlinear terms, into a single uniformly absolutely bounded disturbance function that need to be on-line estimated, and canceled, from the controller. After input gain matrix cancelation, the resulting system is constituted by pure integration (linear) perturbed systems with timevarying additive disturbances. The effects of the unknown disturbance input on the output reconstruction error dynamics (at the observer stage) may be attenuated via a suitable linear combination of iterated injections of the output estimation errors. A set of linear extended observers, here called GPI observers, are subsequently specified which internally model the state dependent additive nonlinearities as time-polynomials of reasonable low orders. The observers' state estimation errors are shown to satisfy a set of decoupled, perturbed, linear differential equations with assignable constant coefficients. The designed observers estimate each individual flat output's associated string of phase variables as well as the time-varying perturbation, or disturbance, input components. Reported results for other applications, such as control of wheeled mobile manipulators (Morales et al., 2014c), control of induction motors (Sira-Ramírez et al., 2013), or the control of combinations of electrical machines and dc-to-dc power converters (Sira-Ramírez and Oliver-Salazar, 2013) encourage the use of GPI control schemes as an alternative of improvement in relation to classic control schemes.

The paper is structured as follows: Section 2 presents the hovercraft model and establishes their flatness property. Additionally, a simplified model is proposed which radically simplifies the disturbance observer and feedback controller design tasks and the problem to be solved is formulated. Section 3 presents the fundamental background results under which the proposed control methodology is established and the generalities about GPI observers. The results obtained are applied to the stabilization and trajectory tracking problem of the hovercraft vessel model. In this section, the state dependent disturbance estimation-disturbance elimination linear output feedback strategy is also developed. Section 4 is devoted to digital computer simulations depicting the performance of the proposed GPI observer-based linear controllers on the hovercraft system under large initial errors, unmodeled unmatched perturbations and model parametric uncertainties. Finally, Section 5 presents the conclusions of the work.

\section{Problem formulation}

\subsection{The hovercraft model and its flatness property}

The equations of motion for a rather general surface vessel dynamics can be written in the following form (Fossen, 1994):

$$
\begin{aligned}
\mathbf{M} \dot{\nu}+\mathbf{C}(\boldsymbol{\nu}) \boldsymbol{\nu}+\mathrm{D} \boldsymbol{\nu} & =\boldsymbol{\tau} \\
\dot{\boldsymbol{\eta}} & =\mathbf{J}(\boldsymbol{\eta}) \boldsymbol{\nu}
\end{aligned}
$$

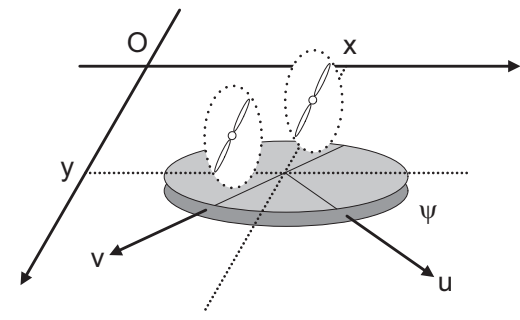

Fig. 1. Simplified hovercraft system.

where

$\mathbf{C}(\boldsymbol{\nu})=\left[\begin{array}{ccc}0 & 0 & -m_{22} v \\ 0 & 0 & m_{11} u \\ m_{22} v & -m_{11} u & 0\end{array}\right], \quad \mathbf{J}(\boldsymbol{\eta})=\left[\begin{array}{ccc}C_{\psi} & -S_{\psi} & 0 \\ S_{\psi} & C_{\psi} & 0 \\ 0 & 0 & 1\end{array}\right]$

with $\mathbf{M}$ being the inertia matrix, $\mathbf{C}(\boldsymbol{\nu}) \boldsymbol{\nu}$ is the vector of Coriolis and centripetal terms and $\mathbf{D} \boldsymbol{\nu}$ is the vector of friction and hydrodynamic damping terms. The vector $\nu=[u, v, r]^{T}$ denotes the vehicle linear velocities in surge, sway and angular velocity in yaw expressed in the vehicle-fixed reference frame. The vector $\boldsymbol{\eta}=[x, y, \psi]^{T}$ expresses the position and orientation in a earth-fixed reference frame and $C_{\psi} \equiv \cos \psi$ and $S_{\psi} \equiv \sin \psi$. The vector $\boldsymbol{\tau}=\left[\tau_{1}, \tau_{2}, \tau_{3}\right]^{T}$ defines the vector of forces acting on the vehicle in surge and sway and the torque on the vehicle acting on the yaw. The matrices $\mathbf{M}$ and $\mathbf{D}$ are, both, diagonal and given by

$\mathbf{M}=\operatorname{diag}\left\{m_{11}, m_{22}, m_{33}\right\}, \quad \mathbf{D}=\operatorname{diag}\left\{d_{11}, d_{22}, d_{33}\right\}$

In this work, we consider the model of the underactuated hovercraft vessel system illustrated in Fig. 1. A mathematical model for such vessel system was derived in Fantoni et al. (2000) taking into consideration the following assumptions:

Assumption 1. The vessel system is assumed to be symmetric with regard to the axes $u$ and $v$, i.e. $m_{11}=m_{22}$.

Assumption 2. The control force acting in sway is zero, i.e. $\tau_{2}=0$.

Assumption 3. The hydrodynamic damping coefficients $d_{11}$ and $d_{33}$ are both zero, i.e. $d_{11}=d_{33}=0$. In the case that these damping terms were actually present, they could be compensated by partial state feedback though the control terms $\tau_{1}$ and $\tau_{3}$.

Finally, the following mathematical model is proposed for the underactuated hovercraft vessel system:

$$
\begin{aligned}
& \dot{x}=u C_{\psi}-v S_{\psi r} \\
& \dot{y}=u S_{\psi}+v C_{\psi} \\
& \dot{\psi}=r \\
& \dot{u}=v r+\tau_{u} \\
& \dot{v}=-u r-\beta v \\
& \dot{r}=\tau_{r}
\end{aligned}
$$

where $\tau_{1}=m_{11} \tau_{u}, \tau_{3}=m_{33} \tau_{r}$ and $\beta=d_{22} / m_{22}$.

The hovercraft model given in (4) is differentially flat, with flat outputs given by the position in earth fixed reference frame $([x, y])$, i.e. all system variables in (4) can be differentially parameterized solely in terms of $x$ and $y$, and a finite number of their time derivatives. Their expressions are as follows:

$$
\begin{aligned}
& \psi=\arctan \left(\frac{\ddot{y}+\beta \dot{y}}{\ddot{x}+\beta \dot{x}}\right) \\
& u=\frac{\dot{x}(\ddot{x}+\beta \dot{x})+\dot{y}(\ddot{y}+\beta \dot{y})}{\sqrt{(\ddot{x}+\beta \dot{x})^{2}+(\ddot{y}+\beta \dot{y})^{2}}} \\
& v=\frac{\dot{y} \ddot{x}-\dot{x} \ddot{y}}{\sqrt{(\ddot{x}+\beta \dot{x})^{2}+(\ddot{y}+\beta \dot{y})^{2}}}
\end{aligned}
$$




$$
\begin{aligned}
r= & \frac{y^{(3)}(\ddot{x}+\beta \dot{x})-x^{(3)}(\ddot{y}+\beta \dot{y})-\beta^{2}(\ddot{x} \dot{y}-\ddot{y} \dot{x})}{(\ddot{x}+\beta \dot{x})^{2}+(\ddot{y}+\beta \dot{y})^{2}} \\
\tau_{u}= & \frac{\ddot{x}(\ddot{x}+\beta \dot{x})+\ddot{y}(\ddot{y}+\beta \dot{y})}{\sqrt{(\ddot{x}+\beta \dot{x})^{2}+(\ddot{y}+\beta \dot{y})^{2}}} \\
\tau_{r}= & \frac{y^{(4)}(\ddot{x}+\beta \dot{x})-x^{(4)}(\ddot{y}+\beta \dot{y})}{(\ddot{x}+\beta \dot{x})^{2}+(\ddot{y}+\beta \dot{y})^{2}}+\frac{\beta\left(y^{(3)} \ddot{x}-x^{(3)} \ddot{y}\right)-\beta^{2}\left(x^{(3)} \dot{y}-y^{(3)} \dot{x}\right)}{(\ddot{x}+\beta \dot{x})^{2}+(\ddot{y}+\beta \dot{y})^{2}} \\
& -2 \frac{y^{(3)}(\ddot{x}+\beta \dot{x})-x^{(3)}(\ddot{y}+\beta \dot{y})-\beta^{2}(\ddot{x} \dot{y}-\ddot{y} \dot{x})}{\left[(\ddot{x}+\beta \dot{x})^{2}+(\ddot{y}+\beta \dot{y})^{2}\right]^{2}} \\
& \times\left[(\ddot{x}+\beta \dot{x})\left(x^{(3)}+\beta \ddot{x}\right)+(\ddot{y}+\beta \dot{y})\left(y^{(3)}+\beta \ddot{y}\right)\right]
\end{aligned}
$$

Proof. Operating with the first two expressions of (4) the following is obtained:

$$
u=\dot{x} C_{\psi}+\dot{y} S_{\psi}
$$$$
v=\dot{y} C_{\psi}-\dot{x} S_{\psi}
$$

Differentiating now the first two expressions and using (6) gives

$\ddot{x}=\dot{u} C_{\psi}-u \dot{\psi} S_{\psi}-\dot{v} S_{\psi}-v \dot{\psi} C_{\psi}=\tau_{u} C_{\psi}+\beta v S_{\psi}$

$\ddot{y}=\dot{u} S_{\psi}+u \dot{\psi} C_{\psi}+\dot{v} C_{\psi}-v \dot{\psi} S_{\psi}=\tau_{u} S_{\psi}-\beta v C_{\psi}$

Now, manipulating expressions (7) yielded

$\beta v=\ddot{x} S_{\psi}-\ddot{y} C_{\psi}$

$\tau_{u}=\ddot{x} C_{\psi}+\ddot{y} S_{\psi}$

Using (6) and (8) and after some algebraic manipulations the following result is achieved:

$\psi=\arctan \left[\frac{\ddot{y}+\beta \dot{y}}{\ddot{x}+\beta \dot{x}}\right]$

Substituting (9) in (6) yields

$u=\frac{\dot{x}(\ddot{x}+\beta \dot{x})+\dot{y}(\ddot{y}+\beta \dot{y})}{\sqrt{(\ddot{x}+\beta \dot{x})^{2}+(\ddot{y}+\beta \dot{y})^{2}}}$

$v=\frac{\dot{y}(\ddot{x}+\beta \dot{x})-\dot{x}(\ddot{y}+\beta \dot{y})}{\sqrt{(\ddot{x}+\beta \dot{x})^{2}+(\ddot{y}+\beta \dot{y})^{2}}}=\frac{\dot{y} \ddot{x}-\dot{x} \ddot{y}}{\sqrt{(\ddot{x}+\beta \dot{x})^{2}+(\ddot{y}+\beta \dot{y})^{2}}}$

Finally, the proof is completed after substituting (9) in the expression of the force input, $\tau_{u}$ obtained in (8) and using the fact that $r=\dot{\psi}$ and $\tau_{r}=\ddot{\psi}$.

The lack of invertibility of the relation between the control input vector $\left[\tau_{u}, \tau_{r}\right]$ and the flat outputs highest derivative depicts that the hovercraft dynamics requires a second-order dynamic extension of the input variable $\tau_{u}$. The following result is achieved:

$$
\begin{aligned}
& \tau_{r}= \frac{-x^{(4)} \sin \psi+y^{(4)} \cos \psi-\beta r \tau_{u}}{\beta u+\tau_{u}} \\
&+\frac{-2 r \dot{\tau}_{u}-2 \beta r^{2} v+\beta^{2} u r+\beta^{3} v}{\beta u+\tau_{u}} \\
& \ddot{\tau}_{u}=x^{(4)} \cos \psi+y^{(4)} \sin \psi+2 \beta u r^{2}+2 \beta^{2} r v-\beta v \tau_{r}+r^{2} \tau_{u}
\end{aligned}
$$

Additionally, after comparing (5) and (11), the following result is obtained:

$\beta u+\tau_{u}=\sqrt{(\ddot{x}+\beta \dot{x})^{2}+(\ddot{y}+\beta \dot{y})^{2}}$

Clearly, we are interested in maneuvers for which this quantity is bounded (which is physically reasonable and natural) and it is also bounded away from zero (which somehow limits the class of desired trajectories).

Assumption 4. We assume that the positive quantity, $\sqrt{(\ddot{x}+\beta \dot{x})^{2}+(\ddot{y}+\beta \dot{y})^{2}}$, is uniformly bounded by a constant for all times, and it is nowhere identically zero along the evolution of the system.

\subsection{Simplified model and problem formulation}

From expressions (5) and (11), it is observed that the highly nonlinear, coupled, nature of the system precludes any practical implementation of an exactly linearizing feedback control approach. The key step in our developments is based on the fact that the flat output dynamics of the hovercraft vessel model may be significantly simplified to the following perturbed, phenomenological, system:

$\left[\begin{array}{l}x^{(4)} \\ y^{(4)}\end{array}\right]=\left[\begin{array}{cc}-\left(\beta u+\tau_{u}\right) \sin \psi+\beta v \cos \psi & \cos \psi \\ \left(\beta u+\tau_{u}\right) \cos \psi+\beta v \sin \psi & \sin \psi\end{array}\right]\left[\begin{array}{c}\tau_{r} \\ \ddot{\tau}_{u}\end{array}\right]+\left[\begin{array}{c}\varphi_{x}(t) \\ \varphi_{y}(t)\end{array}\right]$

where $\boldsymbol{\varphi}(t)=\left[\varphi_{x}(t), \varphi_{y}\right]^{T}(t)$ involves all the nonlinearities affecting the system behavior regarded here as an unknown but uniformly absolutely bounded disturbance input that needs to be on-line estimated by means of an observer and, subsequently, canceled from the simplified system dynamics via feedback in order to regulate the flat output vector, $[x, y]^{T}$, towards the desired reference trajectories $\left[x^{*}(t), y^{*}(t)\right]^{T}$.

A key property of the flat output vector $[x, y]^{T}$ is that it vastly eases the off-line trajectory planning aspects for the system and provides a direct way, which involves no need for solutions of differential equations, to establish the open-loop trajectories of all system variables on the basis of the nominal trajectories for the flat outputs. The flat output vector is devoid of any zero dynamics and completely guarantee total internal stability of the system states and outputs including those outputs which are nonminimum phase (Isidori, 2002). These properties facilitate a unified treatment for both stabilization and tracking tasks, within a common framework. Finally, the formulation of the problem is stated as follows:

Given a desired flat output vector of reference trajectories $\left[x^{*}(t)\right.$, $\left.y^{*}(t)\right]^{T}$, devise a linear multi-input output feedback controller for system (13) so that regardless of the vector of unknown disturbances and coupling nonlinearities, represented by $\varphi(t)=\left[\varphi_{x}(t), \varphi_{y}(t)\right]^{T}$, the flat output vector $[x(t), y(t)]^{T}$ tracks the desired reference flat output vector $\left[x^{*}(t), y^{*}(t)\right]^{T}(t)$ even if in an approximate fashion. This approximate character specifically means that the flat output tracking error vector dynamics, $\mathbf{e}=\left[e_{x}, e_{y}\right]^{T}=\left[x-x^{*}(t), y-y^{*}(t)\right]^{T}$, exhibits a closed loop, predominantly linear, asymptotically stable convergent behavior so that the tracking error trajectories are ultimately confined to a small as desired neighborhood on the origin of the tracking error phase space.

\section{GPI observer based linear controller with disturbance estimation-rejection of the hovercraft vessel system}

\subsection{Background results}

An important difference (and a new type of equivalence) between nonlinear differential equations and the corresponding linear differential equations with injected exogenous time-varying signals is established.

Consider the following system of time-varying nonlinear differential equations:

$\dot{y}=A y+\phi(t, y), \quad y\left(t_{0}\right)=y_{0}, \quad y \in \mathbb{R}^{n}$

where $A$ is an $n \times n$ Hurwitz matrix, and $\phi(t, y)$ is a vector of nonlinearities possibly including exogenous signals. Let $z$ be an $n$-dimensional vector and consider the following linear system 
with an exogenous, time-varying, injection:

$$
\dot{z}=A z+\phi\left(t, y\left(t, y_{0}\right)\right), \quad z(0)=y_{0}+b
$$

where $b$ is a constant. The following remarks are proposed.

Remark 1. The time-varying vector $y\left(t, y_{0}\right)$ trivially satisfies the identity (see Pontryagrin, 1962, Chapter 1, pp. 19):

$\dot{y}(t)=A y(t)+\phi(t, y(t)), \quad y\left(t_{0}\right)=y_{0}$

Remark 2. The linear differential equation for $z$ includes a copy of the term $\phi(t, y)$ particularized for the solution $y=y\left(t, y_{0}\right)$ of the nonlinear differential equation. In itself, for every fixed initial condition, $y_{0}$, the term $\phi\left(t, y\left(t, y_{0}\right)\right)$, is a time function expressed as $\xi(t)=\phi\left(t, y\left(t, y_{0}\right)\right)$.

The error $e(t)=y(t)-z(t)$ evolves which is governed by

$\dot{e}=A e, \quad e(0)=-b$

since $A$ is a Hurwitz matrix then $\lim _{t \rightarrow \infty} e(t)=0 \forall b \in \mathbb{R}^{n}$. Furthermore, if $b$ is set to zero, and the initial conditions for $y$ and $z$ coincide, then $y \equiv z \forall t \geq 0$. The consequence of this simple fact is that the nonlinear system (14) is now viewed as a linear system with an exogenous time-varying injection term, $\xi(t)=\phi(t, y(t))$,

$\dot{z}=A z+\xi(t), \quad z\left(t_{0}\right)=y_{0}$

Then, the systems defined in (14) and (18) are identical in the precise sense that their trajectories are the same over any time interval. Consequently, the distinction between $z$ and $y$ becomes irrelevant. Any pertinent consideration of the system (14) may be examined on the linear trajectory equivalent system (18), viewed now without any ambiguity as

$\dot{y}=A y+\xi(t), \quad y(0)=y_{0}$

which is devoid of the state-dependent nonlinear structure.

Frequently, in practice, the nonlinear function $\phi(t, y)$ is unknown due to the unknown parameter values or due to the complex nonlinearities, or simply due to the presence of unknown exogenous time signals affecting $\phi(t, y)$. Define $\hat{\xi}(t)$ as the estimate of the unknown time function $\xi(t)$ such that the difference $\hat{\xi}(t)-\xi(t)$ is guaranteed to be uniformly absolutely bounded, in norm, by a small positive constant $\epsilon$, i.e.

$\sup _{t}\|\xi(t)-\hat{\xi}(t)\| \leq \epsilon$

then it can be observed that the error vector, $e(t)=y(t)-z(t)$, is ultimately uniformly absolutely bounded in norm by a small positive constant $\delta(\epsilon)$ that depends on the eigenvalue $\lambda$ of $A$ with the largest (negative) real part, i.e. that which is closest to the imaginary axis in the complex plane. The Hurwitzian character of A implies that for every constant, $(n+m) \times(n+m)$, symmetric, positive definite, matrix $Q=Q^{T}>0$, there exists a symmetric, positive definite $(n+m) \times(n+m)$ matrix $P=P^{T}>0$ such that $A^{T} P+P A=-Q$. The Lyapunov function candidate, $V(e)=\frac{1}{2} e^{T} P e$, exhibits a time derivative, along, the solutions of the perturbed error system, $\dot{e}=A e+(\xi(t)-\hat{\xi}(t))$, given by

$$
\begin{aligned}
\dot{V} & =\frac{1}{2} e^{T}\left(A^{T} P+P A\right) e+e^{T} P(\xi(t)-\hat{\xi}(t)) \\
& =-\frac{1}{2} e^{T} Q e+\|P\|\|e\| \epsilon \\
& \leq-\frac{1}{2}\|e\|^{2} \sqrt{\lambda_{\min }\left(Q Q^{T}\right)}+\|P\|\|e\| \epsilon \\
& \leq-\left(\sqrt{\left.\lambda_{\min }\left(A A^{T}\right)\right)}\|P\|\|e\|^{2}+\|P\|\|e\| \epsilon .\right.
\end{aligned}
$$

$\dot{V}(e)$ is negative outside the ball $\mathcal{B}$ defined by

$$
\mathcal{B}=\left\{e \in \mathbb{R}^{n} \mid\|e\| \leq \delta(\epsilon)=\frac{\epsilon}{\sqrt{\lambda_{\min }\left(A A^{T}\right)}}\right\}
$$

and, consequently, all the solution trajectories $e(t)$ approach the ball $\mathcal{B}$ from the outside. Otherwise, they evolve uniformly bounded inside $\mathcal{B}$. Obviously, the more negative the eigenvalues of $A$, the smaller radius of the ball $\mathcal{B}$. The implications of the previous result on the observation, or control, of the nonlinear scalar system are

$y^{(n)}=\psi(t, y) u+\phi\left(t, y, \dot{y}, \ldots, y^{(n-1)}\right)$

where the drift function $\phi\left(t, y, \dot{y}, \ldots, y^{(n-1)}\right)$ is completely unknown while the control input gain $\psi(t, y)$ is assumed to be known. Indeed, an active disturbance rejection control designed on the basis of the equivalent system $y^{(n)}=v+\xi(t)$ which is of the form

$\psi(t, y) u=v=\left[y^{*}(t)\right]^{(n)}-\hat{\xi}(t)-\sum_{i=0}^{n-1} k_{n-i} e_{y}^{(i)}$

for suitably selected constant parameters $k$ yields a tracking error system governed by the perturbed vector equation

$\dot{\chi}=F \chi+g(\xi(t)-\hat{\xi}(t))$

with $\chi=\left[e_{y}, \dot{e}_{y}, \ldots, e_{y}^{(n-1)}\right]$ and $F$ in companion form, with, $g$, defined as a column vector of zeros with a 1 in the last entry.

\subsection{GPI observers}

The unknown perturbation vector of time signals, $\xi(t)=\phi(t, y$, $\left.\dot{y}, \ldots, y^{(n-1)}\right)$, needs to be online estimated and then canceled at the controller stage. This function is observable in the sense of Diop and Fliess (see Isidori and Fliess, 1991 for details). This means, in particular, that if $\xi(t)$ is bestowed with an exact linear model, an exact asymptotic estimation of $\xi(t)$ can be asymptotically estimated with the help of a linear observer subject to nonlinear input injection through the known gain. The linear observer design strategy consist of estimating the perturbation input $\xi(t)$ using an, instantaneous, internal time polynomial model, realized in the form of a chain of integrators of length $p-1$ at the observer stage for a fixed, sufficiently large integer $p$. When forcing the dominantly linear, perturbed output estimation error dynamics to exhibit an asymptotically convergent behavior, the internal model for $\xi(t)$ is automatically and continuously self-updated. As a consequence of this, we may safely assume that $\xi(t)$ and a finite number of its time derivatives, $\xi^{(k)}(t)$, for $k=1,2, \ldots, p$ are uniformly absolutely bounded for some sufficiently large integer $p$. In order to state this precisely, let us use $y_{j}$ to denote an estimate of $y^{(j-1)}$ for $j=1, \ldots, n$. The following general result is obtained:

Theorem 1. The GPI observer-based dynamical feedback controller:

$$
\begin{aligned}
& u=\frac{1}{\psi(t, y)}\left[\left[y^{*}(t)\right]^{(n)}-\sum_{j=0}^{n-1}\left(k_{j}\left[y_{j}-\left(y^{*}(t)\right)^{(j)}\right]\right)-\hat{\xi}(t)\right] \\
& \quad \hat{\xi}(t)=z_{1} \\
& \dot{y}_{1}=y_{2}+\lambda_{p+n-1}\left(y-y_{1}\right) \\
& \dot{y}_{2}=y_{3}+\lambda_{p+n-2}\left(y-y_{1}\right) \\
& \vdots \\
& \dot{y}_{n}=\nu+z_{1}+\lambda_{p}\left(y-y_{1}\right) \\
& \dot{z}_{1}=z_{2}+\lambda_{p-1}\left(y-y_{1}\right) \\
& \vdots \\
& \dot{z}_{p-1}=z_{p}+\lambda_{1}\left(y-y_{1}\right) \\
& \dot{z}_{p}=\lambda_{0}\left(y-y_{1}\right)
\end{aligned}
$$

asymptotically exponentially drives the tracking error phase variables $e_{y}^{(k)}=y^{(k)}-\left[y^{*}\right]^{(k)}, k=0,1, \ldots, n-1$ to an arbitrary small neighborhood of the origin, of the tracking error phase space, which can be made as small as desired from the appropriate choice of the controller gain parameters $\left\{k_{0}, \ldots, k_{n-1}\right\}$. Furthermore, the estimation errors, defined as $\tilde{e}^{(i)}=y^{(i)}-y_{i}, \quad i=0, \ldots, n-1$, and the perturbation 
estimation error, defined as $z_{m}-\xi^{(m-1)}(t), m=1, \ldots, p$, asymptotically exponentially converge towards a small as desired neighborhood of the origin of the reconstruction error space, which can be made as small as desired with the appropriate choice of the controller gain parameters $\left\{\lambda_{0}, \ldots, \lambda_{p+n-1}\right\}$ (see the proof in Sira-Ramírez et al., 2009; Morales et al., 2014c).

Remark 3. The integer $m$, depicting the order of approximation of the lumped disturbance signal $\xi(t)$, is typically chosen as $m=3$ or, at most, $m=5^{1}$ (see Sira-Ramírez et al., 2010 for experimental details).

Remark 4. Finally, in order to avoid, possible, large initial peaking of the observer variables responses, we use a clutch function to smooth these transient peaking responses in all the observer variables that need to be used in the controller. The "clutch" is defined as a time function smoothly increasing from 0 to 1 , during a small time interval $[0, \varepsilon)$. We set, for instance, the smoothing function, $s_{f}(t) \in[0,1]$ and define it in the following (non-unique) way:

$S_{f}(t)= \begin{cases}1 & \text { for } t>\varepsilon \\ \sin ^{q}\left(\frac{\pi t}{2 \varepsilon}\right) & \text { for } t \leq \varepsilon\end{cases}$

where $q$ is a suitably large positive even integer. Then, the "smoothing" of the observer variables may be carried out according with

$F_{j s}(t)=F_{j}(t) s_{f}(t)$

\subsection{GPI observer based linear controller with disturbance estimation-rejection of the hovercraft vessel system}

The fundamental idea in regulating the hovercraft vessel model, or accomplishing a given suitable flat output vector reference trajectory tracking, is to use the simplified, phenomenological model (13). To accomplish this, we primarily estimate, even if in an approximately as desired fashion, the unknown disturbance vector $\boldsymbol{\varphi}(t)$ and, after that, to include a cancelation term of such disturbance effects in the designed input control vector, $\boldsymbol{\tau}(t)=\left[\tau_{r}, \tau_{u}\right]$, and then asymptotically impose a linear stable closed loop dynamics by appropriately feeding back the estimated time derivatives of the measured flat output vector $[x(t), y(t)]^{T}$.

A GPI observer is considered including a reasonable, selfupdating, instantaneous time-polynomial model ${ }^{2}$ for each unknown component, state dependent, disturbance input vector $\boldsymbol{\varphi}(t)$. For this internal model, we use for each component of $\varphi(t)$ an unspecified element of a third degree family of time-polynomials, denoted by $\boldsymbol{\varphi}_{1}^{(4)}(t)=\left[\varphi_{1 x}^{(4)}, \varphi_{1 y}^{(4)}\right]^{T}=\mathbf{0}$. Then, the GPI observer based flat output feedback controller is conceived as follows:

$$
\left[\begin{array}{c}
\tau_{r} \\
\ddot{\tau}_{u}
\end{array}\right]=\left[\begin{array}{cc}
-\frac{\sin \psi}{\beta u+\tau_{u}} & \frac{\cos \psi}{\beta u+\tau_{u}} \\
\frac{\beta v \sin \psi}{\beta u+\tau_{u}}+\cos \psi & -\frac{\beta v \cos \psi}{\beta u+\tau_{u}}+\sin \psi
\end{array}\right]\left[\begin{array}{l}
\nu_{x} \\
\nu_{y}
\end{array}\right]
$$

with

$\nu_{x}=-\hat{\varphi}_{1 x s}+\left[x^{*}(t)\right]^{(4)}-\sum_{i=0}^{3} k_{i}^{x}\left(\hat{x}_{s}^{(i)}-\left[x^{*}(t)\right]^{(i)}\right)$

\footnotetext{
${ }^{1}$ As a justification for such a low degree, recall John von Neumann's statement: "With four parameters I can fit an elephant, and with five I can make him wiggle his trunk!"

${ }^{2}$ Also known as Taylor Polynomial Model.
}

$$
\nu_{y}=-\hat{\varphi}_{1 y s}+\left[y^{*}(t)\right]^{(4)}-\sum_{i=0}^{3} k_{i}^{y}\left(\hat{y}_{s}^{(i)}-\left[y^{*}(t)\right]^{(i)}\right)
$$

where the quantities $\hat{\boldsymbol{\varphi}}_{1 s}=\left[\hat{\varphi}_{1 x s}, \hat{\varphi}_{1 y s}\right]^{T}, \hat{x}_{s}^{(i)}$, and $\hat{y}_{s}^{(i)}$ are smoothing clutch functions to smoothing transient peaking responses in the observer variables used in the control algorithm. Furthermore, the variables $\hat{x}^{(j)}=x_{j}$ and $\hat{y}^{(j)}=y_{j}, j=0,1,2,3$, are generated by

$$
\begin{aligned}
& \dot{x}_{0}=x_{1}+\lambda_{6}^{x}\left(x-x_{0}\right) \\
& \dot{x}_{1}=x_{2}+\lambda_{5}^{x}\left(x-x_{0}\right) \\
& \dot{x}_{2}=x_{3}+\lambda_{4}^{x}\left(x-x_{0}\right) \\
& \dot{x}_{3}=\tau_{r}\left[-\left(\beta u+\tau_{u}\right) \sin \psi+\beta v \cos \psi\right]+\ddot{\tau}_{u} \cos \psi+\varphi_{1 x}+\lambda_{3}^{x}\left(x-x_{0}\right) \\
& \dot{\varphi}_{1 x}=\varphi_{2 x}+\lambda_{2}^{x}\left(x-x_{0}\right) \\
& \dot{\varphi}_{2 x}=\varphi_{3 x}+\lambda_{1}^{x}\left(x-x_{0}\right) \\
& \dot{\varphi}_{3 x}=\lambda_{0}^{x}\left(x-x_{0}\right) \\
& \dot{y}_{0}=y_{1}+\lambda_{6}^{y}\left(y-y_{0}\right) \\
& \dot{y}_{1}=y_{2}+\lambda_{5}^{y}\left(y-y_{0}\right) \\
& \dot{y}_{2}=y_{3}+\lambda_{4}^{y}\left(y-y_{0}\right) \\
& \dot{y}_{3}=\tau_{r}\left[\left(\beta u+\tau_{u}\right) \cos \psi+\beta v \sin \psi\right]+\ddot{\tau}_{u} \sin \psi+\varphi_{1 y}+\lambda_{3}^{y}\left(y-y_{0}\right) \\
& \dot{\varphi}_{1 y}=\varphi_{2 y}+\lambda_{2}^{y}\left(y-y_{0}\right) \\
& \dot{\varphi}_{2 y}=\varphi_{3 y}+\lambda_{1}^{y}\left(y-y_{0}\right) \\
& \dot{\varphi}_{3 y}=\lambda_{0}^{y}\left(y-y_{0}\right)
\end{aligned}
$$

The estimation error vector, $\tilde{\mathbf{e}}=\left[\tilde{e}_{x}, \tilde{e}_{y}\right]^{T}$, with $\tilde{e}_{x}=x-x_{0}$ and $\tilde{e}_{y}=y-y_{0}$, evolves according with the following linear perturbed dynamics:

$\tilde{e}_{x}^{(7)}+\lambda_{6}^{x} \tilde{e}_{x}^{(6)}+\cdots+\lambda_{1}^{x} \dot{\tilde{e}}_{x}+\lambda_{0}^{x} \tilde{e}_{x}=\varphi_{x}^{(4)}(t)$

$\tilde{e}_{y}^{(7)}+\lambda_{6}^{y} \tilde{e}_{y}^{(6)}+\cdots+\lambda_{1}^{y} \dot{\tilde{e}}_{y}+\lambda_{0}^{y} \tilde{e}_{y}=\varphi_{y}^{(4)}(t)$

Clearly, if $\varphi_{x}^{(4)}(t)$ and $\varphi_{y}^{(4)}(t)$ are uniformly absolutely bounded, and if the choice of the coefficients $\left\{\lambda_{6}^{x}, \ldots, \lambda_{0}^{x}\right\}$ and $\left\{\lambda_{6}^{y}, \ldots, \lambda_{0}^{y}\right\}$ is made in such a way that the roots of the dominant characteristic polynomials

$\hat{p}_{x}(s)=s^{7}+\lambda_{6}^{x} s^{6}+\cdots+\lambda_{1}^{x} s+\lambda_{0}^{x}$
$\hat{p}_{y}(s)=s^{7}+\lambda_{6}^{y} s^{6}+\cdots+\lambda_{1}^{y} s+\lambda_{0}^{y}$

have all their roots sufficiently far from the imaginary axis, in the left half of the complex plane. The observer parameters were chosen so as to obtain the following desired closed-loop characteristic polynomial:

$p_{\text {obs }}(s)=\left(s^{2}+2 \zeta_{o} \omega_{n o} s+\omega_{n o}^{2}\right)^{3}\left(s+p_{o}\right)$

where $\omega_{n o}, \zeta_{o}$ and $p_{o}$ are positive quantities. Therefore, the coefficients $\left\{\lambda_{6}^{x}, \ldots, \lambda_{0}^{x}\right\}$ and $\left\{\lambda_{6}^{y}, \ldots, \lambda_{0}^{y}\right\}$ are given by

$\lambda_{6}^{x}=\lambda_{6}^{y}=p_{o}+6 \zeta_{o} \omega_{n o}$

$\lambda_{5}^{x}=\lambda_{5}^{y}=12 \zeta_{0}^{2} \omega_{n o}^{2}+6 \zeta_{0} \omega_{n o} p_{o}+3 \omega_{n o}^{2}$

$\lambda_{4}^{x}=\lambda_{4}^{y}=12 \zeta_{o} \omega_{n o}^{3}+12 \zeta_{o}^{2} \omega_{n o}^{2} p_{o}+8 \zeta_{o}^{3} \omega_{n o}^{3}+3 \omega_{n o}^{2} p_{o}$

$\lambda_{3}^{x}=\lambda_{3}^{y}=8 \zeta_{0}^{3} \omega_{\text {no }}^{3} p_{o}+12 \zeta_{o}^{2} \omega_{n o}^{4}+12 \zeta_{o} \omega_{n o}^{3} p_{o}+3 \omega_{n o}^{4}$

$\lambda_{2}^{x}=\lambda_{2}^{y}=6 \zeta_{o} \omega_{n o}^{5}+12 \zeta_{o}^{2} \omega_{n o}^{4} p_{o}+3 \omega_{n o}^{4} p_{o}$

$\lambda_{1}^{x}=\lambda_{1}^{y}=6 \zeta_{o} \omega_{n o}^{5} p_{o}+\omega_{n o}^{6}$

$\lambda_{0}^{x}=\lambda_{0}^{y}=\omega_{\text {no }}^{6} p_{o}$

Then, the trajectories of the estimation errors, $\tilde{e}_{x}$ and $\tilde{e}_{y}$, and of their time derivatives, converge to a small neighborhood of the origin of the phase space of the observer estimation error. The further away the roots are located into the left half of the complex plane, the smaller the radius of the disk representing the neighborhood around the origin of the estimation error phase space.

On the other hand, the closed loop tracking error vector $\mathbf{e}=\left[e_{x}, e_{y}\right]^{T}$, with $e_{x}=x-x^{*}(t)$, and $e_{y}=y-y^{*}$, satisfies the 
following predominantly linear dynamics:

$$
\begin{aligned}
& e_{x}^{(4)}+k_{3}^{x} e_{x}^{(3)}+k_{2}^{x} \ddot{e}_{x}+k_{1}^{x} \dot{e}_{x}+k_{0}^{x} e_{x}=\varphi_{x}(t)-\hat{\varphi}_{x}(t)+\varrho_{x}(t) \\
& e_{y}^{(4)}+k_{3}^{y} e_{y}^{(3)}+k_{2}^{y} \ddot{e}_{y}+k_{1}^{y} \dot{e}_{y}+k_{0}^{y} e_{y}=\varphi_{y}(t)-\hat{\varphi}_{y}(t)+Q_{y}(t)
\end{aligned}
$$

where $\boldsymbol{\varrho}(t)=\left[Q_{x}(t), Q_{y}(t)\right]^{T}$ in expression (38), depicts the effect of the small flat output phase variables estimation errors, generated by the observer, and the effects of the disturbance signal, on-line, estimation errors and the differences $\varphi_{x}(t)-\hat{\varphi}_{x}$ and $\varphi_{y}(t)-\hat{\varphi}_{y}$, produce reference trajectory tracking errors $e_{x}=x-x^{*}(t)$ and $e_{y}=y-y^{*}(t)$, that also asymptotically exponentially converges towards a small vicinity of the origin of the tracking error space. The design coefficients $k_{i}^{x}$ and $k_{i}^{y}$ are chosen so as to render the closed loop characteristic polynomials:

$p_{x}(s)=s^{4}+k_{3}^{x} s^{3}+k_{2}^{x} s^{2}+k_{1}^{x} s+k_{0}^{x}$

$p_{y}(s)=s^{4}+k_{3}^{y} s^{2}+k_{2}^{y} s^{2}+k_{1}^{y} s+k_{0}^{y}$

into the Hurwitz polynomials with desirable roots. The gains $\left\{k_{3}^{x}, \ldots, k_{0}^{x}\right\}$ and $\left\{k_{3}^{y}, \ldots, k_{0}^{y}\right\}$ of the closed loop characteristic polynomial were determined by a term by term comparison with the following desired Hurwitz polynomial:

$p_{d c}(s)=\left(s^{2}+2 \zeta_{c} \omega_{n c} s+\omega_{n c}^{2}\right)^{3}$

where $\omega_{n c}$ and $\zeta_{c}$ are positive quantities. The gains $\left\{k_{3}^{x}, \ldots, k_{0}^{x}\right\}$ and $\left\{k_{3}^{y}, \ldots, k_{0}^{y}\right\}$ of the GPI controllers were then set to be

$k_{3}^{\chi}=k_{3}^{y}=4 \zeta_{c} \omega_{n c}$

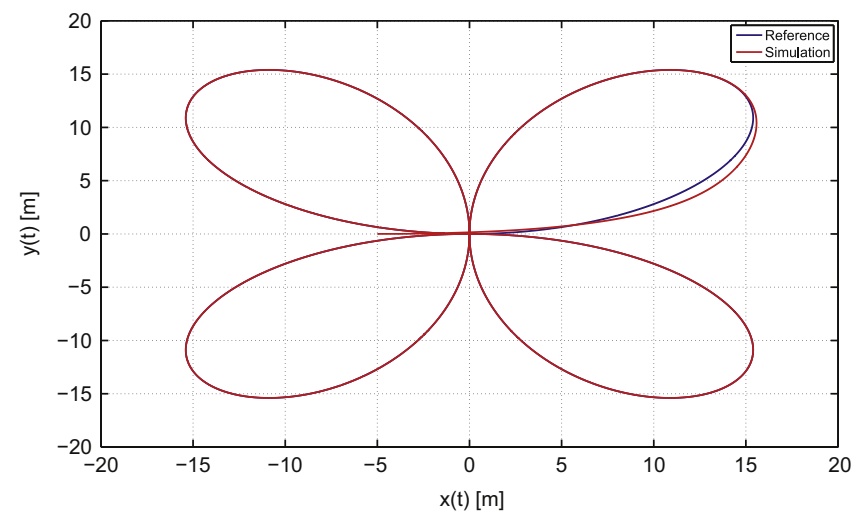

Fig. 2. Four-folium trajectory tracking. Hovercraft reference and simulation paths.
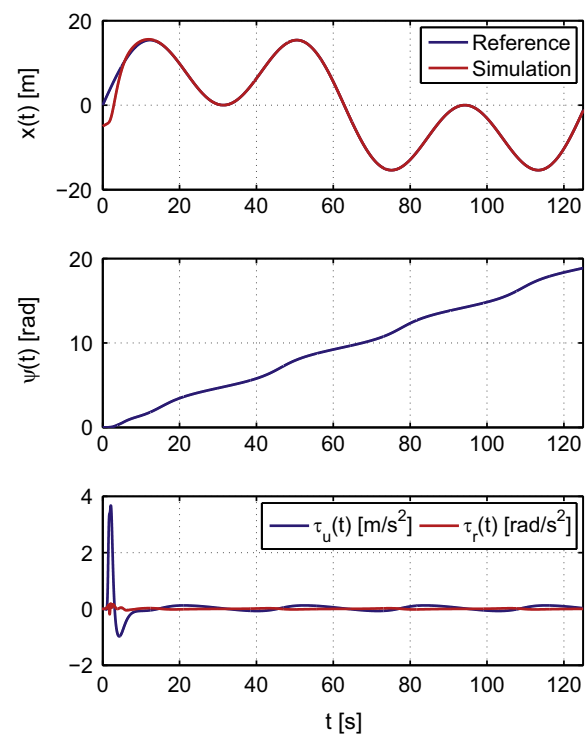

$k_{2}^{x}=k_{2}^{y}=4 \zeta_{c}^{2} \omega_{n c}^{2}+2 \omega_{n c}^{2}$

$k_{1}^{x}=k_{1}^{y}=4 \zeta_{c} \omega_{n c}^{3}$

$k_{0}^{x}=k_{0}^{y}=\omega_{n c}^{4}$

It is intuitively clear that the closed loop dynamics in expression (39) is less severely affected by the uncertainties than the corresponding dynamics of the observer estimation errors. This fact results in smaller magnitudes of the feedback gains $k_{i}^{x}$ and $k_{i}^{y}$, than those used for the design of the GPI observers. Finally, we have the following result:

Proposition 1. Given a smooth vector of desired reference trajectories for the components of the flat output vector, $\left[x^{*}(t), y^{*}(t)\right]^{T}$, and provided the observers and the controllers constant gains appearing in (35) and (39), are chosen so that the roots of the corresponding closed loop characteristic polynomials are chosen deep into the left half of the complex plane (according to expressions (37) and (41)), then the GPI observer based linear feedback controllers given by Eqs. (32)-(33), produce a set of perturbed closed loop flat outputs tracking error dynamics whose trajectories converge, in an asymptotically exponentially dominated manner, to a small as desired neighborhood of the origins of the flat outputs tracking error phase spaces. Moreover, the flat output phase variables estimation errors satisfy linear perturbed dynamics whose trajectories also dominantly converge in an asymptotically exponentially dominated manner to small as desired neighborhoods of the origins of the reconstruction errors phase spaces. As a result, the disturbance vector components of $\boldsymbol{\varphi}(t)=\left[\varphi_{x}(t), \varphi_{y}(t)\right]^{T}$ are closely estimated with an error bounded by a small as desired neighborhood of zero. As the location of the roots of the dominating characteristic polynomials are further pushed into the left half of the complex plane, the tighter around the origin are all these tracking, or estimation, bounding neighborhoods.

\section{Simulation results}

Numerical simulations were carried out in order to verify that the above designed controller performs very well in terms of quick convergence of the tracking errors to a small neighborhood of zero, smooth transient responses, low control effort, and robustness in the following cases: (i) large initial errors, (ii) un-modeled unmatched perturbations, and (iii) model parametric uncertainties. In all of the following simulations, the trajectory proposed is a "four-folium" trajectory, defined in the earth fixed coordinate
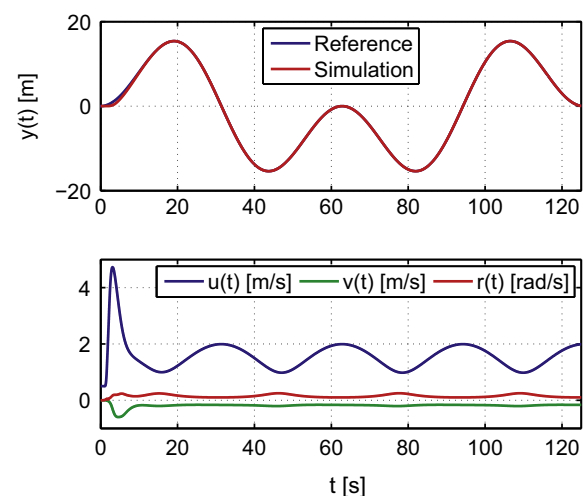

$\mathrm{t}[\mathrm{s}]$

Fig. 3. Four-folium trajectory tracking. Feedback controlled angular orientation, velocity variables and applied inputs. 
frame, and centered around the origin. Under these assumptions, the nominal trajectories of the flat outputs are specified as

$x^{*}(t)=\rho\left(\sin \left(A_{1} t\right)+\sin \left(A_{2} t\right)\right)$

$y^{*}(t)=\rho\left(\cos \left(A_{1} t\right)-\cos \left(A_{2} t\right)\right)$

In the simulations, the only system parameter $\beta$ was set to be $\beta=1.2$ and the parameters which define the "four-folium" path were selected as $\rho=10 \mathrm{~m}, A_{1}=0.05 \mathrm{rad} / \mathrm{s}$ and $A_{2}=0.15 \mathrm{rad} / \mathrm{s}$. The hovercraft starts in position and orientation in the earth fixed coordinates of $x(0)=-5 \mathrm{~m}, y(0)=0 \mathrm{~m}, \psi(0)=0$ rad, with velocities $[u(0), v(0), r(0)]=[0.5,0,0]$. The gains of the GPI observers were designed with the help of the following dominating Hurwitz characteristic polynomial $\left(s^{2}+2 \zeta_{0} \omega_{n o} s+\omega_{n o}^{2}\right)^{3}\left(s+p_{o}\right)$ with $\zeta_{o}=1$, $\omega_{n o}=10$ and $p_{o}=10$, while the gains of the feedback controllers were set according to the polynomial $\left(s^{2}+2 \zeta_{c} \omega_{n c} s+\omega_{n c}^{2}\right)^{2}$ with $\zeta_{c}=1, \omega_{n c}=0.7$. The time $\varepsilon$ where the clutching functions are applied was set to $\varepsilon=2 \mathrm{~s}$. The time sampling used in the simulations is set as $1 \times 10^{-3} \mathrm{~s}$.

\subsection{Tracking a four-folium trajectory}

In this section, the computer simulation is carried out under ideal conditions, which implies that there is no un-modeled external perturbation force and the only system parameter is perfectly known. Fig. 2 depicts the controlled evolution of the

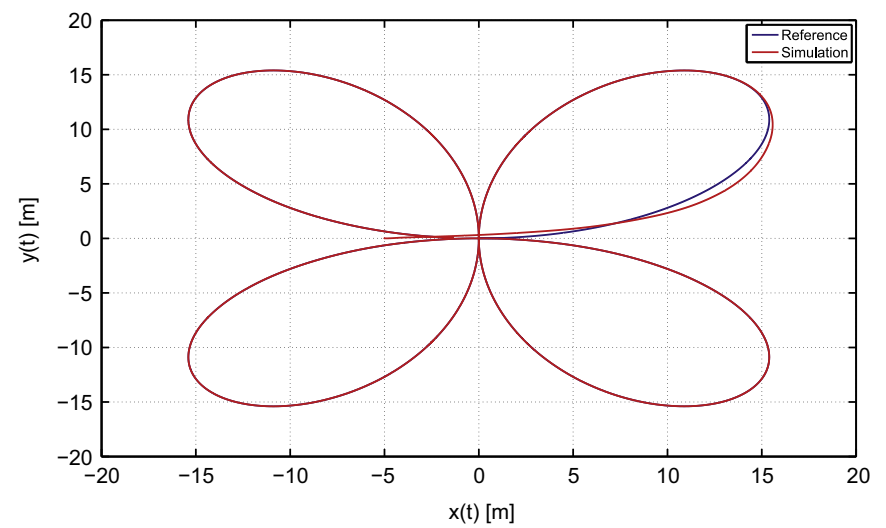

Fig. 4. Four-folium trajectory tracking under unmodeled unmatched perturbations. Hovercraft reference and simulation paths.
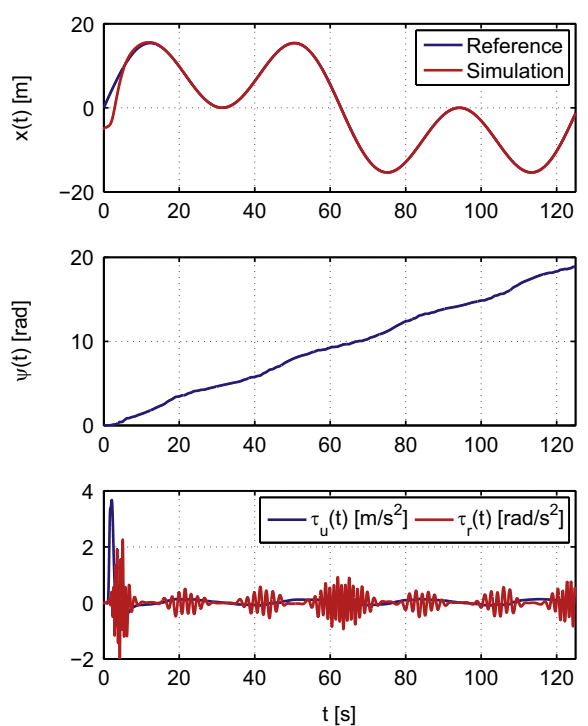

hovercraft position coordinates when the vessel motions are started significantly far away from the desired trajectory. Fig. 3 illustrates the closed loop trajectories for the state and control input variables of the hovercraft system.

\subsection{Robustness with respect to un-modeled unmatched perturbations}

We present some results concerning the robustness properties of the proposed controller. Specifically, we introduced in the nonactuated dynamics (i.e. in the sway acceleration equation) an unmodeled external perturbation force, simulating a "wave field" effect of the form

$\dot{v}=-u r-\beta v+\lambda(x(t))$

$\lambda(x(t))=L\left[\sin (f x(t))+\frac{1}{5} \cos \pi f x(t)\right]$

with $L=0.1$ and $f=1$. Figs. 4 and 5 show the path tracking and the closed loop trajectories for the state and control variables under the un-modeled sustained perturbations. In spite of the unmatched nature of the perturbation signal, the proposed GPI observer based linear controller, with the same controller parameters used in the previous section, efficiently corrects the undesirable effects of the perturbation input forces.

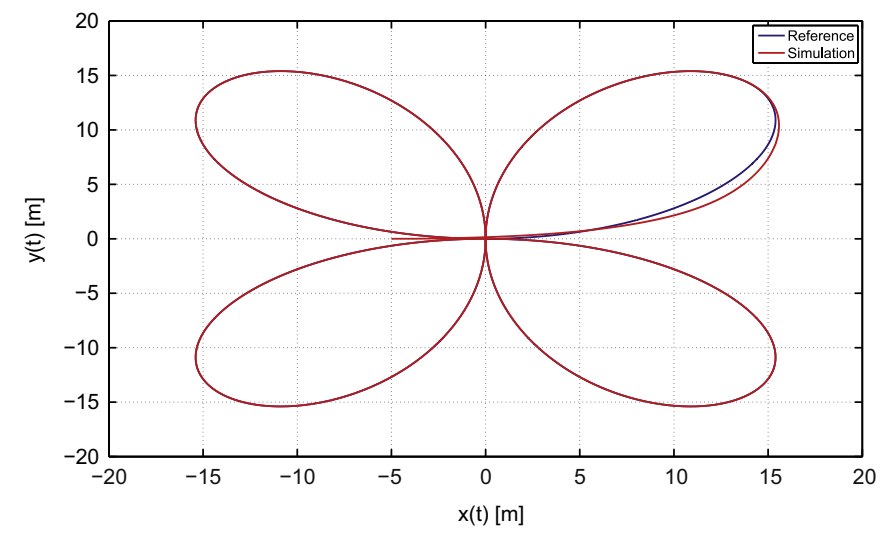

Fig. 6. Four-folium trajectory tracking with controller gain mismatches. Hovercraft reference and simulation paths.
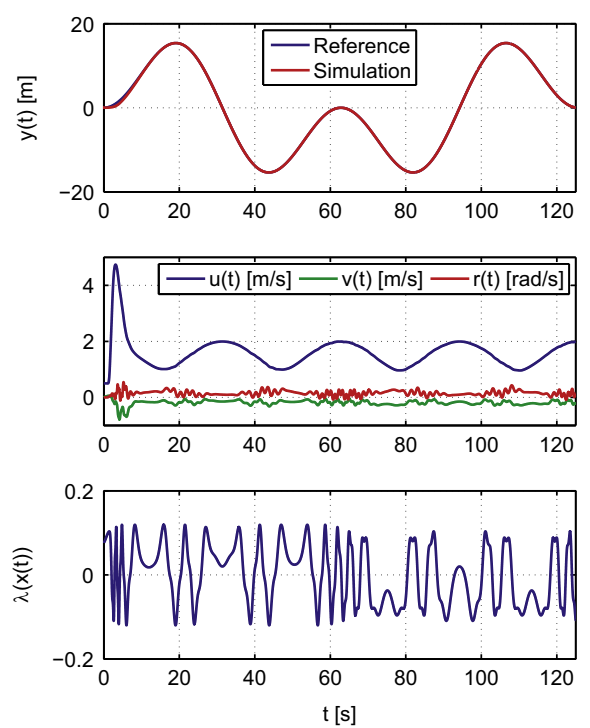

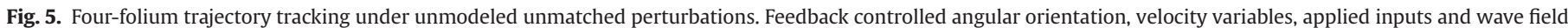
effect. 

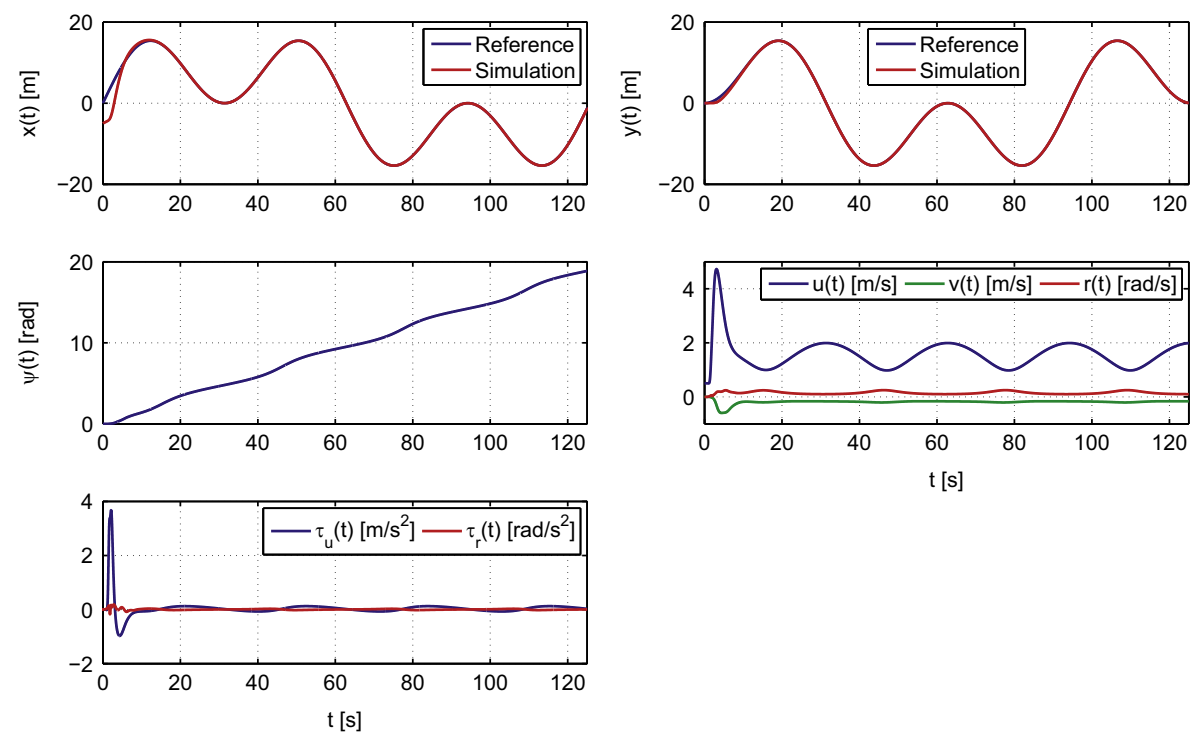

Fig. 7. Four-folium trajectory tracking with controller gain mismatches. Feedback controlled angular orientation, velocity variables and applied inputs.

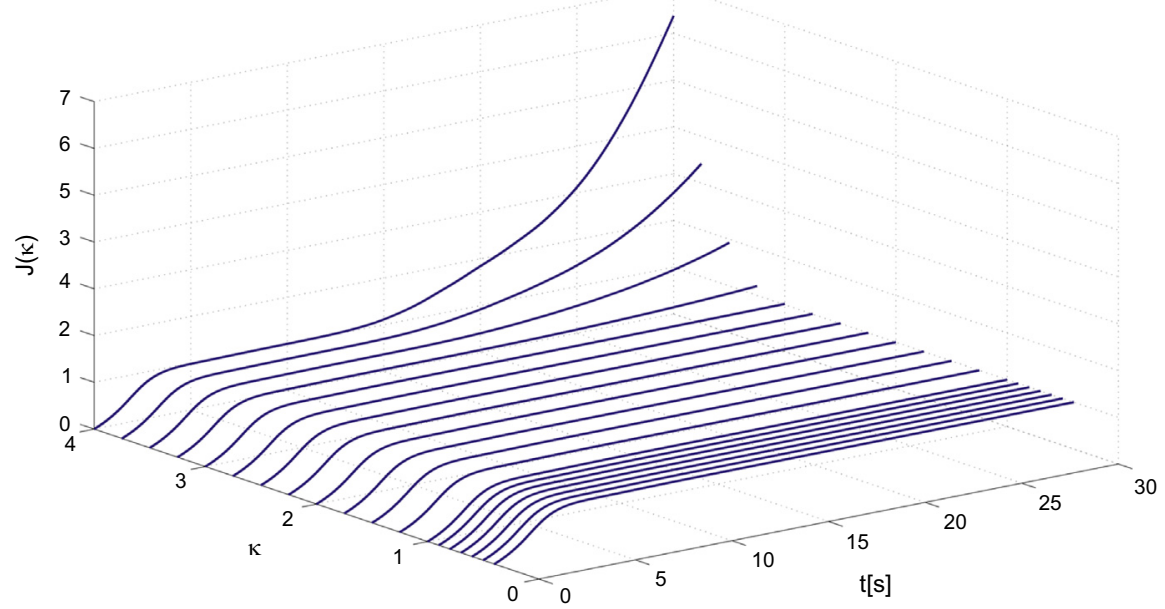

Fig. 8. Normalized function $J(\kappa)$ for different values of the estimated controller value $\beta_{c}=\kappa \beta$.

\subsection{Robustness with respect to controller gain mismatches}

We simulated the behavior of the closed loop system when the parameter $\beta$ is not precisely known and the controller is implemented with an estimated value $\beta_{c}$. Specifically, we conducted simulations in which an error of the order of $20 \%$ of the parameter $\beta$ is assumed (i.e. $\beta_{c}=1.2 \beta$ ). Figs. 6 and 7 illustrate the results of the simulation performed. As one may observe, although there are modeling errors, the feedback controller corrects the motion of the hovercraft, guides the errors of the states to a small neighborhood of zero and compensates for the errors in the model parameters as well.

Additionally, in order to investigate the robustness of the proposed control system, we carried out a study on the effects of the range of variation of the estimated value $\beta_{c}$ in the controller, set as $\beta_{c}=\kappa \beta$ with $\kappa$ defined as a real positive constant. We developed a series of simulations where the value of $\beta_{c}$, was made to vary from $\beta_{c}=0.36$ (i.e. $30 \%$ of the actual $\beta$ value $(\kappa=0.3)$ ) to $\beta_{c}=4.8$ (i.e. $400 \%$ of the actual $\beta$ value $(\kappa=4)$ ). A normalized criteria was included to evaluate the performance of the proposed controller which is defined as

$J(\kappa)=\frac{1}{\mu} \int_{0}^{\infty}\left(e_{x}^{2}(\kappa)+e_{y}^{2}(\kappa)\right) d t$ where $e_{x}(\kappa)$ and $e_{y}(\kappa)$ denote the trajectory tracking errors of the earth coordinate position variables for a particular value of $\kappa$ and $\mu$ is defined by the following expression:

$\mu=\lim _{t \rightarrow \infty} \int_{0}^{t}\left(e_{x}^{2}+e_{y}^{2}\right) d t \quad$ with $\kappa=1$

The performance of the proposed controller for different values of $\kappa$ is depicted in Fig. 8. For $\kappa=0.3$ the controller produces an unstable response of the system. For $0.4 \leq \kappa \leq 3$ the value of (44) became constant when the time grows up. This implies that the controller is working successfully, the tracking errors of the states evolute to small neighborhoods of zero and, consequently, the hovercraft vessel system tracks the path accurately. Finally, when $\kappa>3$ the behavior of the controller starts deteriorating, giving a value of (44) that is not constant when the time grows up. In conclusion, these results demonstrate that the proposed output feedback control method is robust with respect to a wide range of uncertainty on the only model parameter, $\beta_{c}$, to be specified in the controller.

\section{Conclusions}

In this paper, we have illustrated, within the context of the trajectory tracking problem in the highly nonlinear, multi-variable, 
hovercraft vessel system, the use of approximate, yet accurate, state-dependent disturbance estimation, and simultaneous state estimation, via linear Generalized Proportional (GPI) observers aided by a linear output controller. The direct cancelation of the unknown perturbation inputs, via the linearizing feedback law, considerably simplifies the ultimate feedback controller design to a linear feedback scheme for each flat output evolution. Finally, simulations were provided where the robustness of the proposed control method is assessed with respect to large initial errors, unmodeled unmatched perturbations and parametric uncertainties in the model.

In future work, different experimental branches are the focus or our attention. They are detailed as follows: (a) development a real hovercraft laboratory prototype and (b) synthesization of the proposed robust controller in a real platform. In this case, several algorithms based on the GPI philosophy have been carried out in electronic applications such as the control of Buck converters (Zurita-Bustamante et al., 2011) or the control of single-phase multilevel cascade inverters (Juárez-Abad et al., 2014). Taking into consideration the slow dynamics of the hovercraft vessel model in comparison with the electronic applications described above, the synthesization of the robust controller designed on a field programmable gate array (FPGA) (Juárez-Abad et al., 2014; Morales et al., 2014a, 2013; Zurita-Bustamante et al., 2011) or low-cost architectures based on reconfigurable logic (Morales et al., 2014b) does not present any problem regarding computation time requirements.

Behal, A., Dawson, D.M., Dixon, W.E., Fang, Y., 2002. Tracking and regulation control of an underactuated surface vessel with nonintegrable dynamics. IEEE Trans. Automat. Control 47 (3), 495-500.

Brockett, R.W., 1983. Asymptotic stability and feedback linearization. In: Differential Geometric Control Theory. pp. 181-191.

Fantoni, I., Lozano, R., Mazenc, F., Pettersen, K.Y., 2000. Stabilization of a nonlinear underactuated hovercraft. Int. J. Robust Nonlinear Control 10 (8), 645-654.

Fliess, M., Join, C., 2009. Model-free control and intelligent PID controllers: towards a possible trivialization of nonlinear control. In: 15th IFAC Symposium on System Identification, Saint-Malo, France. pp. 1531-1550.

Fliess, M., Lévine, J., Martin, Ph., Rouchon, P., 1995. Flatness and defect of nonlinear systems: introductory theory and examples. Int. J. Control 61 (6), 1327-1361.

Fliess, M., Marquez, R., Delaleau, E., Sira-Ramírez, H., 2002. Correcteurs proportionnels integraux Généralisés. ESAIM: Control Optim. Calc. Var. 7, 23-41.

Fossen, T.I., 1994. Guidance and Control of Ocean Vehicles. John Wiley and Sons Ltd., UK, ISBN: 0-471-94113-1.

Fossen, T.I., 2011. Handbook of Marine Craft Hydrodynamics and Motion Control. John Wiley and Sons Ltd., UK, ISBN: 9781119991496.

Isidori, A., 2002. Nonlinear Control Systems, 3rd ed. Springer Verlag, London.

Isidori, S., Fliess, M., 1991. Nonlinear observability, identifiability and persistent trajectories. In: 36th IEEE Conference on Decision and Control, Brighton, UK.

Juárez-Abad, J.A., Linares-Flores, J., Guzmán-Ramírez, E., Sira-Ramírez, H., 2014. Generalized proportional integral tracking controller for a single-phase multilevel cascade inverter: an FPGA implementation. IEEE Trans. Ind. Inf. 10 (1), 256-266.

Kaminer, I., Pascoal, A., Hallberg, E., Silvestre, C., 1998. Trajectory tracking for autonomous vehicles: an integrated approach for guidance and control. J. Guid. Control Dyn. 21 (1), 29-38.

Lebefer, E., Petersen, K.Y., Nijmeijer, H., 2003. Tracking control of an underactuated ship. IEEE Trans. Control Syst. Technol. 11 (1), 52-61.

Morales, R., Somolinos, J.A., Morón, C., García, A., 2013. Space-state robust control of a buck converter with amorphous core coil and variable load. Measurement 46, 3863-3870.

Morales, R., Somolinos, J.A., Sira-Ramírez, H., 2014a. Control of a DC motor using algebraic derivative estimations with real-time experiments. Measurement 47, 401-417.

Morales, R., Rinón, F., Dondo Gazzano, J., López, J.C., 2014b. Real-time algebraic derivative estimations using a novel low cost architecture based on reconfigurable logic. Sensors 14, 9349-9368.

Morales, R., Sira-Ramírez, H., Somolinos, J.A., Robust control of underactuated wheeled mobile manipulators using GPI disturbance observers. Multibody Syst Dyn., vol. 32(4), pp. 511-533, 2014c.

Petersen, K.Y., Egeland, O., 1996. Exponential stabilization on an underactuated surface vessel. In: Proceedings 35th IEEE Conference on Decision Control, Kobe Japan, December 1996. pp. 967-971.

Pettersen, K.Y., Nijmeijer, H., 1998. Global practical stabilization and tracking for an underactuated ship-a combined averaging and backstepping approach. In: Proceedings IFAC Conference on System Structure and Control, Nantes, France July 1998. pp. 59-64.

Pontryagrin, L.S., 1962. Ordinary Differential Equations. Addison-Wesley, Reading, MA.

Prasanth Kumar, R., Dasgupta, A., Kumar, C.S., 2005. Real-time optimal motion planning for autonomous underwater vehicles. Ocean Eng. 32, 1431-1447.

Prasanth Kumar, R., Dasgupta, A., Kumar, C.S., 2007. Robust trajectory control of underwater vehicles using time delay control law. Ocean Eng. 34, 842-849.

Repoulias, F., Papadopoulos, E., 2007. Planar trajectory planning and tracking control design for underactuated AUVs. Ocean Eng. 34, 1650-1667.

Reyhanoglou, M., 1997. Exponential stabilization on an underactuated autonomous surface vessel. Automatica 33 (12), 2249-2254.

Sira-Ramírez, H., 2002. Dynamic second-order sliding mode control of the hovercraft vessel. IEEE Trans. Control Syst. Technol. 10 (6), 860-865.

Sira-Ramírez, H., Agrawal, S., 2004. Differentially Flat Systems. Marcel Dekker Inc., New York, NY.

Sira-Ramírez, H., Aguilar, C., 2000. On the control of the Hovercraft system. Dyn. Control 10, 151-163.

Sira-Ramírez, H., Oliver-Salazar, M.A., 2013. On the robust control of BuckConverter DC-motor combinations. IEEE Trans. Power Electron. 28 (8), 3912-3922.

Sira-Ramírez, H., Núñez, C., Visairo, N., 2009. Robust sigma-delta generalized proportional integral observer based control of a buck converter with uncertain loads. Int. J. Control 83 (8), 1631-1640.

Sira-Ramírez, H., Ramírez-Neria, M., Rodríguez-Angeles, A., 2010. On the linear control of nonlinear mechanical systems. In: 49th IEEE Conference on Decision and Control, Atlanta, Georgia, USA. pp. 1999-2004.

Sira-Ramírez, H., González-Montanez, F., Cortés-Romero, J.A., 2013. A robust linear field-oriented voltage control for the induction motor: experimental results. IEEE Trans. Ind. Electron. 60 (8), 3025-3033.

Wondergem, M., Lefeber, E., Pettersen, K.Y., Nijmeijer, H., 2011. Output feedback tracking of ships. IEEE Trans. Control Syst. Technol. 19 (2), 442-448.

Yuh, J., 2000. Design and control of autonomous underwater robots: a survey. Int. J. Control 8, 7-24.

Zurita-Bustamante, E.W., Linares-Flores, J., Guzmán-Ramírez, E., Sira-Ramírez, H., 2011. A comparison between the GPI and PID controllers for the stabilization of a DC-DC "Buck" converter: a field programmable gate array implementation. IEEE Trans. Ind. Electron. 58 (11), 5251-5261. 